Pacific Journal of Mathematics

A MEASURE THEORETICAL PROOF OF THE
CONNES-WOODS THEOREM ON AT-FLOWS 


\title{
A MEASURE THEORETICAL PROOF OF THE CONNES-WOODS THEOREM ON AT-FLOWS
}

\section{TOSHIHIRO HAMACHI}

\begin{abstract}
It was shown by A. Connes and J. Woods that every ITPFI factor of type III 0 is characterized to be an AFD factor whose flow of weights is conservative, aperiodic, and approximately transitive (AT). In this paper, a measure theoretical proof of their result will be shown from the side of ergodic theory, without using modular theory.
\end{abstract}

1. Introduction. ITPFI factors, introduced by Araki and Woods [1], provide us concrete models of von Neumann algebras. Among approximately finite dimensional (AFD) factors of type III $_{0}$, their exact position was characterized by Connes and Woods [1], whose result says the flow of weights associated with an ITPFI factor is conservative, aperiodic, and approximately transitive (AT), and conversely. As every ITPFI factor is the Krieger factor arising from a product odometer action with a product measure, and as the isomorphic classes of AFD factors of type III $_{0}$ correspond bijectively with the orbit equivalence classes of ergodic amenable actions of type III $_{0}$ by countable groups of non-singular transformations, their result in effect says that an ergodic amenable action of type III $_{0}$ by a countable group of nonsingular transformations is orbit equivalent with a product odometer action if and only if its associated flow is conservative, aperiodic, and AT (see Definition 17). In such a measure theoretical setting, the one direction that product odometer action implies AT was proved directly by Hawkins [6].

In this paper we would like to present a purely measure theoretical proof of the other direction, which seems to be more difficult. The proof is based on the following observations. Given a countable group $G$ of type III $_{0}$, ergodic, non-singular transformations, a transformation group $\mathscr{G}$ is introduced $(\S 3)$. $\mathscr{G}$ is orbit equivalent with $G$ when the action of $G$ is amenable. $\mathscr{G}$ is equipped with all the information available from the AT-property of the associated flow of $G$, and it is easier to check that $\mathscr{G}$ is a product odometer action rather than to check $G$. Our approach might be helpful for the reader not familar with modular theory of von Neumann algebras, and the notion of 
comparison of finite weights used in the Connes-Woods argument will become clear through our measure theoretical presentation.

The content is the following. Functions $f_{g} \in \mathbf{L}^{1}(X)_{+}$and measures $\psi_{g}\left(g \in[\mathscr{G}]_{*}^{\nu}\right)$ are introduced (see Definition 10$)$, which give a link between the AT-property and product odometer action. We will prepare Lemmas 11-16. Lemma 13 which corresponds to Lemma 5.9 and Lemma 6.4 in [2], is a critical point of the proof ( $\S 3)$. Also we need a characterization of an ergodic amenable action of type III $_{0}$ by a countable group of non-singular transformations which is orbit equivalent with a product odometer $(\S 2)$. This says that any multiple tower with constant Jacobian is refined by a tower with constant Jacobian relative to a modified measure which is close to the original measure (Proposition 7 and Corollary 19). In fact this notion is very closely related to a characterization obtained by Katznelson (Theorem 6.6 in [7]), but is slightly different (see Remark 8 and Corollary 19). Also we note that this corresponds with the product property (Definition 7.1 [2]), which is a variation of Størmer's property of being "asymptotically a product state [10]."

The author thanks T. Girodano and J. Woods for helpful discussions of various points in this paper. He also thanks the referees for suggesting several improvements.

2. Preliminaries. Let $G$ be an ergodic countable group of nonsingular transformations on a Lebesgue space $(\Omega, \mathscr{B}, m)$, where $m$ is a $\sigma$-finite measure.

For $\omega \in \Omega$, we denote the orbit $\{g \omega ; g \in G\}$ by $\operatorname{Orb}_{G}(\omega)$. The group $\left\{\phi ; \phi\right.$ a non-singular transformation such that $\phi \omega \in \operatorname{Orb}_{G}(\omega)$ a.e. $\omega\}$ is denoted by $[G]$ and called the full group of $G$. By a partial transformation $\phi$ we mean a pair of measurable subsets $\mathscr{D}(\phi)$ and $\mathscr{I}(\phi)$ and a measurable bijection from $\mathscr{D}(\phi)$ to $\mathscr{I}(\phi)$ satisfying that $\phi \omega \in \operatorname{Orb}_{G}(\omega)$, a.e. $\omega \in \mathscr{D}(\phi)([3])$. The sets $\mathscr{D}(\phi)$ and $\mathscr{I}(\phi)$ are said to be $G$-Hopf equivalent. We denote the set of all partial transformations by $[G]_{*}$ and the set $\left\{\phi \in[G]_{*} ; m(\mathscr{D}(\phi))<\right.$ $\infty, m(\mathscr{I}(\phi))<\infty\}$ by $[G]_{*}^{m}$ respectively. We note that $[G]_{*}^{m}$ plays the same role as the predual of a von Neumann algebra.

Definition 1. A tower of $G$ is a finite collection $\zeta=\left\{e_{\alpha, \beta} \in\right.$ $\left.[G]_{*}, \alpha, \beta \in \Lambda\right\}$ of partial transformations satisfying the following conditions:

(a) $E_{\beta}=\mathscr{D}\left(e_{\beta, \beta}\right)$ are disjoint.

(b) $e_{\alpha, \beta} e_{\beta, \gamma}=e_{\alpha, \gamma}, \alpha, \beta, \gamma \in \Lambda$. 
Notice that for fixed $\beta$ the sets $\mathscr{D}\left(e_{\alpha, \beta}\right)$ and $\mathscr{I}\left(e_{\beta, \alpha}\right)$ are all equal to $E_{\beta}$. We call $E_{\beta}$ a floor of $\zeta$. We denote the set $\bigcup_{\beta \in \Lambda} E_{\beta}$ by $s(\zeta)$ and call it the support of $\zeta$. We also denote the set $\left\{e_{\alpha, \beta} \omega ; \alpha \in \Lambda\right\}$ for $\omega \in E_{\beta}$ by $\operatorname{Orb}_{\zeta}(\omega)$ and call it the orbit of $\zeta$. A finite union of towers with disjoint supports is called a multiple tower.

Definition 2. Let $\zeta=\left\{e_{\alpha, \beta} ; \alpha, \beta \in \Lambda\right\}$ be a tower. A measurable subset $E \subset s(\zeta)$ is said to be $\zeta$-adapted if

$$
e_{\beta, \alpha}\left(E_{\alpha} \cap E\right)=E_{\beta} \cap E
$$

for $\alpha$ and $\beta \in \Lambda$ with $m\left(E_{\alpha} \cap E\right)>0$ and $m\left(E_{\beta} \cap E\right)>0$.

In particular, $E$ is said to be $\zeta$-invariant if

$$
\operatorname{Orb}_{\zeta}(\omega) \in E \text {, a.e. } \omega \in E \text {. }
$$

When we restrict each partial transformation $e_{\alpha, \beta}$ to a $\zeta$-adapted set $E$, we obtain the restriction of the tower $\zeta$ to $E$ and denote it by $\left.\zeta\right|_{E}$.

Definition 3. We say that a tower $\zeta=\left\{e_{\alpha, \beta} ; \alpha, \beta \in \Lambda\right\}$ has a constant $Q$-Jacobian if each Radon-Nikodym derivative $\left(d Q e_{\beta, \alpha} / d Q\right)(\omega)$ is constant on $E_{\alpha}$ where $Q$ a finite measure on $s(\zeta)$ which is equivalent with $m$. We call the vector $\left(\left(d Q e_{\beta, \alpha} / d Q\right)(\omega) ; \beta \in \Lambda\right)$ a distribution of $\zeta$ relative to $Q$. As $Q$ is determined by the restriction $\nu$ of $Q$ on $E_{\alpha}$ and the distribution $\mathbf{q}=\left(\left(d Q e_{\beta, \alpha} / d Q\right) ; \beta \in \Lambda\right)$, we sometimes denote $Q$ by $\nu_{\mathbf{q}}$.

Definition 4. Let $\sum_{i=1}^{n} \oplus \zeta_{i}$ be a multiple tower with $\zeta_{i}=\left\{e_{\alpha, \beta}^{i}\right.$; $\left.\alpha, \beta \in \Lambda_{i}\right\}, \Gamma_{i}, 1 \leq i \leq n$, finite sets and $\zeta=\left(e_{\varepsilon, \delta} ; \varepsilon, \delta \in \Lambda\right)$ a tower with

$$
\Lambda=\left\{(i, \alpha, r) ; 1 \leq i \leq n, \alpha \in \Lambda_{i}, r \in \Gamma_{i}\right\} .
$$

If

$$
\begin{aligned}
& \bigcup_{i=1}^{n} s\left(\zeta_{i}\right)=s(\zeta), \\
& \bigcup_{\gamma \in \Gamma_{i}} E_{i \alpha r}=E_{\alpha}^{i}, \quad \alpha \in \Lambda_{i}, \quad\left(E_{\alpha}^{i} \text { a floor of } \zeta_{i}\right), \\
& e_{i \alpha r, i \beta r} \omega=e_{\alpha, \beta}^{i} \omega \text { a.e. } \omega \in E_{i \beta r}, \quad i \alpha r, i \beta r \in \Lambda,
\end{aligned}
$$

then $\sum_{i=1}^{n} \oplus \zeta_{i}$ is said to be refined by $\zeta$.

Definition 5. Let $\zeta=\left\{e_{\alpha, \beta}: \alpha \in \Lambda\right\}$ be a tower, $\varepsilon \in \Lambda$, and $\eta=\left\{e_{r, s} ; r, s \in \Gamma\right\}$ a tower with the support $E_{\varepsilon}$. Then $\zeta$ is refined 
by a tower

$$
\xi=\left\{e_{\alpha r, \beta s} ; \alpha r, \beta s \in \Lambda \times \Gamma\right\}
$$

satisfying the following condition that for all $\alpha, \beta \in \Lambda$ and $r, s \in \Gamma$,

$$
\begin{aligned}
E_{\alpha} & =\bigcup_{t \in \Gamma} E_{\alpha t}, \\
e_{\varepsilon s, \alpha s} & =e_{\varepsilon, \alpha} \omega, \quad \omega \in E_{\alpha s}, \\
e_{\varepsilon r, \varepsilon s} \omega & =e_{r, s} \omega, \quad \omega \in E_{\varepsilon s} \quad \text { and } \\
e_{\alpha r, \beta s} \omega & =e_{\alpha r, \varepsilon r} e_{\varepsilon r, \varepsilon s} e_{\varepsilon s, \beta s} \omega, \quad \omega \in E_{\beta s} .
\end{aligned}
$$

$\xi$ is called a product to the towers $\zeta$ and $\eta$ and denoted by $\xi=\zeta \otimes \eta$.

By an amenable action of a group $G$, we will mean a countable group of non-singular transformations admitting a non-singular transformation $T$ satisfying $[G]=[T]$, where $[T]=\left[\left\{T^{i} ; i \in \mathbf{Z}\right\}\right]$.

It is known ([8]) that the action of $G$ is amenable if and only if there exists a sequence of product towers $\zeta_{1} \otimes \zeta_{2} \otimes \cdots \otimes \zeta_{n}, n=1,2, \ldots$, such that

$$
\bigcup_{n \geq 1} \operatorname{Orb}_{\zeta_{1} \otimes \cdots \otimes \zeta_{n}}(\omega)=\operatorname{Orb}_{G}(\omega)<\text { a.e. } \omega
$$

and that

$$
\bigvee_{n=1}^{\infty} \mathscr{B}\left(\zeta_{1} \otimes \cdots \otimes \zeta_{n}\right)=\mathscr{B} .
$$

Here $\mathscr{B}(\zeta)$ means the sub $\sigma$-algebra generated by all floors of a tower $\zeta$. Hence, we see that the action of $G$ is amenable if and only if for any $\varepsilon>0$, and any finite collection of partial transformations $g_{1}, \ldots, g_{n} \in[G]_{*}^{m}$ there exists a tower $\zeta$ satisfying the following conditions (a) and (b):

(a) $\mathscr{D}\left(g_{i}\right), \mathscr{I}\left(g_{i}\right) \in^{m, \varepsilon} \mathscr{B}(\zeta)$.

Here $\epsilon^{m, \varepsilon}$ means the set in its left-hand side is $\varepsilon$-approximated by a set in $\mathscr{B}(\zeta)$ in the sense of $m$-measure symmetric difference.

(b) $m\left(\omega \in \mathscr{D}\left(g_{i}\right) ; g_{i} \omega \in \operatorname{Orb}_{\zeta}(\omega)\right)>(1-\varepsilon) m\left(\mathscr{D}\left(g_{i}\right)\right), 1 \leq i \leq n$.

Take an infinite product space $\Omega=\prod_{n=1}^{\infty}\left\{0,1, \ldots, r_{n}-1\right\}$ $\left(r_{n} \in \mathbf{N}\right)$ and an infinite product measure $m=\prod_{n=1}^{\infty} m_{n}, m_{n}$ a probability measure with $m_{n}(\varepsilon)>0,0 \leq \varepsilon \leq r_{n}-1$. Let $G_{n}$ be the finite group consisting of all bijective transformations acting on the product space $\prod_{i=1}^{n}\left\{0,1, \ldots, r_{i}-1\right\}, n \geq 1$. Each group $G_{n}$ naturally can act on $\Omega$ by fixing all coordinates after $n$. Putting $G=\bigcup_{n=1}^{\infty} G_{n}$, we call $G$ a product odometer action with a product measure. We will show a characterization of such an action in the following proposition. 
Proposition 6. An ergodic countable group $G$ of non-singular transformations on $(\Omega, \mathscr{B}, m)$ is orbit equivalent with a product odometer action with a product measure if and only if for any $\varepsilon>0$, and for any finite collection of partial transformations $g_{1}, \ldots, g_{n} \in[G]_{*}^{m}$, there exist a finite measure $P \sim m$ and a tower $\zeta$ with constant $P$-Jacobian satisfying

$$
\begin{aligned}
& \mathscr{D}\left(g_{i}\right), \mathscr{I}\left(g_{i}\right) \in^{m, \varepsilon} \mathscr{B}(\zeta), \\
& m\left(\omega \in \mathscr{D}\left(g_{i}\right) \cap s(\zeta) ; g_{i} \omega \in \operatorname{Orb}_{\zeta}(\omega)\right)>(1-\varepsilon) m\left(\mathscr{D}\left(g_{i}\right)\right),
\end{aligned}
$$

and

$$
\|P-m\|_{s(\zeta) \cap E}<\varepsilon,
$$

where $E=\bigcup_{i}\left(\mathscr{D}\left(g_{i}\right) \cup \mathscr{I}\left(g_{i}\right)\right)$ and $\|P-m\|_{A}=\int_{A}\left|1-\frac{d P}{d m}\right| d m$.

Proof. (If part.) If $G=\bigcup_{n=1}^{\infty} G_{n}$ is a product of odometer action with a product measure on $(\Omega, m)=\left(\prod_{n=1}^{\infty}\left\{0,1, \ldots, r_{n}-1\right\}\right.$, $\left.\prod_{n=1}^{\infty} m_{n}\right)$ then each $g_{n} \in G_{n}$ has constant $m$-Jacobian on each cylinder set determined by the first $n$ coordinates.

For $n \geq 1$, consider a tower $\zeta_{n}=\left\{e_{\alpha, \beta} ; \alpha, \beta \in \prod_{i=1}^{n}\{0,1, \ldots\right.$, $\left.\left.r_{i}-1\right\}\right\}$ with constant $m$-Jacobian defined by

$$
\begin{aligned}
E_{\beta} & =\left[\beta_{1}, \ldots, \beta_{n}\right]_{1}^{n} \text { (cylinder set), and } \\
e_{\alpha, \beta} \omega & =\left(\alpha_{1}, \ldots, \alpha_{n}, \omega_{n+1}, \omega_{n+2}, \ldots\right), \quad \text { for } \omega=\left(\omega_{i}\right)_{i \geq 1} \in E_{\beta},
\end{aligned}
$$

where $\alpha=\alpha_{1} \cdots \alpha_{n}$. Each $g \in[G]_{*}$ moves only finitely many coordinates depending on $\omega \in \Omega$. So, given a finite number of $g_{1}, \ldots, g_{k} \in$ $[G]_{*}$, each $g_{i} \omega$ is in $\operatorname{Orb}_{\zeta_{n}}(\omega)$ except on a small subset of the domain $\mathscr{D}\left(g_{i}\right)$ if $n$ is sufficiently large. The domains $\mathscr{D}\left(g_{i}\right)$ and the images $\mathscr{F}\left(g_{i}\right), 1 \leq i \leq k$, can be approximated by a finite union of cylinder sets $E_{\beta}$ in the sense of measure symmetric difference if $n$ is sufficiently large.

(Only if part.) We may suppose $m(\Omega)<\infty$. Obviously, our condition implies that the action of $G$ is amenable. So, there exists a non-singular transformation $T$ such that $[T]=[G]$. Each of orbit equivalence classes of amenable and ergodic actions of type $\mathrm{I}_{n}$, $1 \leq n \leq \infty, \mathrm{II}_{1}$, and $\mathrm{II}_{\infty}$ is unique and these are orbit equivalent with product odometer actions with a product measure ([8]). So, we may assume that the action of $G$ is of type III. Take a sequence $\left(A_{n}\right)_{n \geq 1}$ of measurable sets which are dense in $\mathscr{B}$ and each element of which appears infinitely often in the sequence. Take $\varepsilon_{n}>0$ such that $\sum_{n=1}^{\infty} \varepsilon_{n}<m(\Omega)$. We will show by an inductive argument the existence of a decreasing sequence of sets $\left(H_{k}\right)_{k \geq 1}$, where $\Omega=H_{1}$, a 
sequence of measures $Q_{k} \sim m$ on $H_{k}$ and a sequence of towers $\zeta_{k}$ satisfying the following conditions (a)-(e):

(a) $\zeta_{k}$ has a constant $Q_{k}$-Jacobian and $s\left(\zeta_{k}\right)=H_{k}$.

(b) $H_{k}$ is $\zeta_{k-1}$-invariant, and $\zeta_{k}$ is a refinement of the restriction $\left.\zeta_{k-1}\right|_{H_{k}}$ in a product form, i.e., $\zeta_{k}=\left.\zeta_{k-1}\right|_{H_{k}} \otimes \eta_{k}$, and

$$
\begin{aligned}
& \frac{d Q_{k-1} e_{\alpha, \beta}}{d Q_{k-1}}(\omega)=\frac{d Q_{k} e_{\alpha \gamma, \beta \gamma}}{d Q_{k}}(\omega), \quad \omega \in E_{\beta \gamma}, \\
& \quad \text { for } e_{\alpha, \beta} \in \zeta_{k-1} \text { and } e_{\alpha \gamma, \beta \gamma} \in \zeta_{k} .
\end{aligned}
$$

(c) $m\left(H_{k-1} \backslash H_{k}\right)<\varepsilon_{k}$, and $\exp \left(-\varepsilon_{k}\right)<\frac{d Q_{k}}{d Q_{k-1}}(\omega)<\exp \left(\varepsilon_{k}\right), \omega \in H_{k}$.

(d) $A_{i} \cap H \in^{m, \varepsilon_{k}} \mathscr{B}\left(\zeta_{k}\right), 1 \leq i \leq k$.

(e) $m\left(\omega \in H_{k} ; T_{H_{k}} \omega \in \operatorname{Orb}_{\zeta_{k}}(\omega)\right)>\left(1-\varepsilon_{k}\right) m\left(H_{k}\right)$, where $T_{H_{k}}$ is the induced transformation of $T$ on the set $H_{k}$.

We may assume $A_{1}=\Omega$ and let $\zeta_{1}$ be the trivial tower and $Q_{1}=$ $m$. Now suppose that we have measurable sets $H_{1} \supset H_{2} \supset \cdots \supset H_{n}$, measures $Q_{1}, Q_{2}, \ldots, Q_{n}$ and towers $\zeta_{1}, \zeta_{2}, \ldots, \zeta_{n}$ satisfying the above conditions $(a)-(e)$, where

$$
\begin{aligned}
\zeta_{i} & =\eta_{1} \otimes \eta_{2} \otimes \cdots \otimes \eta_{i} \\
& =\left\{e_{\varepsilon_{1} \cdots \varepsilon_{i}, \delta_{1} \cdots \delta_{\imath}} ; \varepsilon_{1} \cdots \varepsilon_{i}, \delta_{1} \cdots \delta_{i} \in \prod_{j=1}^{i} \Lambda_{j}\right\} .
\end{aligned}
$$

Choose and fix an $\alpha=\alpha_{1} \cdots \alpha_{n} \in \prod_{i=1}^{n} \Lambda_{i}$. Let $\omega \in H_{n}, \beta=\beta_{1} \cdots \beta_{n}$ and $\gamma=\gamma_{1} \cdots \gamma_{n} \in \prod_{j=1}^{n} \Lambda_{j}$ with $\omega \in E_{\gamma}$ and $T_{H_{n}} \omega \in E_{\beta}$. Then we obtain a $j \in \mathbf{Z}$ satisfying

$$
T_{H_{n}} \omega=e_{\beta, \alpha} T_{E_{\alpha}}^{j} e_{\alpha, \gamma} \omega .
$$

Let us choose arbitrary $\theta>0$ and $N \in \mathbf{N}$. Applying the sufficient condition in the proposition for $\theta, N$ and the partial transformations $T_{E_{\alpha}}^{j},-N \leq j \leq N$, and id $\left.\right|_{e_{\alpha, \beta}}\left(E_{\beta} \cap A_{k}\right)$, we obtain a finite measure $Q \sim m$ and a tower $\eta_{n+1}$ with $s\left(\eta_{n+1}\right) \subset E_{\alpha}$ having a constant $Q$ Jacobian satisfying

$$
\begin{aligned}
& e_{\alpha, \beta}\left(E_{\beta} \cap A_{k}\right) \stackrel{m, \theta}{\epsilon} \mathscr{B}\left(\eta_{n+1}\right), \quad 1 \leq k \leq n+1, \quad \beta \in \prod_{i=1}^{n} \Lambda_{i}, \\
& m\left(\omega \in E_{\alpha} ; T_{E_{\alpha}}^{j} \omega \in \operatorname{Orb}_{\eta_{n+1}}(\omega)\right)>(1-\theta) m\left(E_{\alpha}\right), \quad-N \leq j \leq N . \\
& \exp (-\theta)<\frac{d Q}{d Q_{n}}(\omega)<\exp (\theta), \quad \omega \in s\left(\eta_{n+1}\right),
\end{aligned}
$$


and

$$
m\left(E_{\alpha} \backslash s\left(\eta_{n+1}\right)\right)<\theta .
$$

Now we put $H_{n+1}=\operatorname{Orb}_{\zeta_{n}}\left(s\left(\eta_{n+1}\right)\right)$, and get the product tower $\zeta_{n+1}=\left.\zeta_{n}\right|_{H_{n+1}} \otimes \eta_{n+1}$. Define the finite measure $Q_{n+1}$ by

$$
Q_{n+1}=Q_{\mathbf{q}} \text { on } H_{n+1}
$$

where $\mathbf{q}=\left(\left(d Q_{n} e_{\beta, \alpha} / d Q_{n}\right) ; \beta \in \prod_{i=1}^{n} \Lambda_{i}\right)$. Then it is easy to see $H_{n+1}, Q_{n+1}, \zeta_{n+1}$ satisfy (a)-(e), if $\theta>0$ and $N \in \mathbf{N}$ are chosen small enough and large enough respectively. We see that the set $H=$ $\bigcap_{k=1}^{\infty} H_{k}$ has positive measure. Then for a.e. $\omega \in H$, and for all but a finite number of $k \geq 1$, we obtain $T_{H} \omega=T_{H_{k}} \omega$. Applying Borel Cantelli's lemma for the condition (e), we have that for a.e. $\omega \in H$ and for all but a finite number of $k \geq 1$,

$$
T_{H} \omega=T_{H_{k}} \omega \in \operatorname{Orb}_{\zeta_{k}}(\omega) .
$$

By the condition (c) one can define a positive bounded measurable function $f(\omega)$ on $H$ by

$$
f(\omega)=\prod_{k=2}^{\infty} \frac{d Q_{k}}{d Q_{k-1}}(\omega), \quad \omega \in H .
$$

Define the finite measure $\mu \sim m$ on $H$ by

$$
d \mu(\omega)=\frac{f(\omega) d m(\omega)}{\int_{H} f d m}, \quad \omega \in H,
$$

and the map $\Phi: H \mapsto \prod_{i=1}^{\infty} \Lambda_{i}$ by

$$
\Phi(\omega)=\left(\varepsilon_{i}\right)_{i \geq 1}, \quad \text { for } \omega \in \bigcap_{k \geq 1} E_{\varepsilon_{1} \cdots \varepsilon_{k}} .
$$

Then it follows from (d) and (b) that $\Phi$ produces an invertible and measure preserving orbit equivalence map between $\left[T_{H}\right]$ on $(H$, $\mathscr{B} \cap H, \mu)$ and the product odometer action with a product measure on $\left(\prod_{n=1}^{\infty} \Lambda_{n}, \prod_{n=1}^{\infty} \nu_{n}\right)$ defined by

$$
\nu_{n}\left(\varepsilon_{n}\right)=\sum_{\varepsilon_{1} \cdots \varepsilon_{n-1} \in \prod_{i=1}^{n-1} \Lambda_{\imath}} \mu\left(E_{\varepsilon_{1} \cdots \varepsilon_{n-1} \varepsilon_{n}}\right), \quad \varepsilon_{n} \in \Lambda_{n} .
$$

Since $\mu \sim m$ on $H$ and $T$ is of type III, $T$ is orbit equivalent with $T_{H}$. 
Proposition 7. Let $G$ be an ergodic countable group of non-singular transformations on $(\Omega, \mathscr{B}, m)$ and suppose that $G$ satisfies the following conditions (a) and (b) :

(a) The action of $G$ is amenable.

(b) There exist for any multiple tower $\sum_{i=1}^{n} \bigoplus \zeta_{i}$ with constant $P$ Jacobian ( $P$ a finite measure equivalent with $m$ ), a finite measure $Q \sim$ $m$ and a tower $\zeta$ with constant $Q$-Jacobian which refines $\sum_{i=1}^{n} \oplus \zeta_{i}$ and satisfies $\|P-Q\|_{\bigcup_{i=1}^{n} s\left(\zeta_{1}\right)}<\varepsilon$.

Then $G$ is orbit equivalent with a product odometer action with a product measure.

Proof. It is enough to check the necessary and sufficient condition in Proposition 6. Choose and fix $\varepsilon>0$ and $g_{1}, \ldots, g_{n} \in[G]_{*}^{m}$. Since the action by $G$ is amenable, there exists a tower $\zeta=\left\{e_{\alpha, \beta} ; \alpha, \beta \in\right.$ $\Lambda$ \} such that

$$
\mathscr{D}\left(g_{i}\right), \mathscr{I}\left(g_{i}\right) \stackrel{m, \varepsilon}{\epsilon} \mathscr{B}(\zeta)
$$

and

$$
\begin{aligned}
m\left(\omega \in \mathscr{D}\left(g_{i}\right) \cap s(\zeta) ; g_{i} \omega \in \operatorname{Orb}_{\zeta}(\omega)\right)>(1-\varepsilon) m\left(\mathscr{D}\left(g_{i}\right)\right) & \\
1 & \leq i \leq n .
\end{aligned}
$$

Take an arbitrary floor $E_{\alpha}$ of $\zeta$ and decompose it into a finite number of disjoint sets $A_{j}, 0 \leq j \leq N$, such that

$$
c_{\beta, j} \exp (-\varepsilon)<\frac{d m e_{\beta, \alpha}}{d m}(\omega)<c_{\beta, j} \exp (\varepsilon), \quad \text { a.e. } \omega \in A_{j}
$$

for $\beta \in \Lambda, 1 \leq j \leq N$, where $c_{\alpha, j}=1$, and

$$
m\left(\operatorname{Orb}_{\zeta}\left(A_{0}\right)\right)<\varepsilon \text {. }
$$

Define the measure $P$ by

$$
\begin{aligned}
& P\left(e_{\beta, \alpha} E\right)=c_{\beta, j} m(E), \quad E \subset A_{j}, \quad \text { and } \\
& P\left(e_{\beta, \alpha} E\right)=m(E), \quad E \subset A_{0},
\end{aligned}
$$

restrict $\zeta$ to $\operatorname{Orb}_{\zeta}\left(A_{j}\right)$ and denote the restriction by $\zeta_{j}, 1 \leq j \leq N$. Then $\sum_{j=1}^{N} \bigoplus \zeta_{j}$ has a constant $P$-Jacobian. The condition (b) implies there exist a finite measure $Q \sim P$ and a tower $\xi$ with constant $Q$-Jacobian such that $\xi$ refines $\sum_{j=1}^{N} \bigoplus \zeta_{j}$ and that $\|Q-P\|_{\bigcup_{j=1}^{N} s\left(\zeta_{j}\right)}<\varepsilon$. Thus $\xi$ and $Q$ satisfy the condition in Proposition 6. 
Remark 8. The sufficient condition in Proposition 7 for an ergodic countable group $G$ of non-singular transformations to be orbit equivalent with a product odometer action with a product measure is in fact a necessary condition. This will be proved in the next section (Corollary 19).

3. Transformation group $\mathscr{G}$. Let $G$ be a type III $_{0}$ countable ergodic group of non-singular transformations on $(\Omega, \mathscr{B}, m)$. On the product space $(\Omega \times \mathbf{R}, \mathscr{B} \otimes \mathscr{B}(\mathbf{R}))$ with the product measure $d \nu(\omega, u)$ $=d m(\omega) \exp (u) d u$, each $g \in G$ produces a skew product transformation $\tilde{g}$ defined by

$$
\tilde{g}(\omega, u)=\left(g \omega, u-\log \left(\frac{d m g}{d m}(\omega)\right)\right), \quad(\omega, u) \in \Omega \times \mathbf{R},
$$

which is $\nu$-preserving and commutes with the flow $T_{t}(\omega, u)=$ $(\omega, u+t), t \in \mathbf{R}$. Here $\mathscr{B}(\mathbf{R})$ denotes the $\sigma$-algebra of all Lebesgue measurable subsets of $\mathbf{R}$. By $\mathscr{F}$, we denote the sub $\sigma$-algebra consisting of all $\widetilde{G}$-invariant sets, where $\widetilde{G}=\{\tilde{g} ; g \in G\}$. By $X$, we denote the quotient space $\Omega \times \mathbf{R} / \mathscr{F}$, that is, the space of all $\widetilde{G}$-ergodic components. Let $\pi$ be the natural projection $\Omega \times \mathbf{R} \mapsto X$. Take an arbitrary $\sigma$-finite measure $\mu$ on $X$ which is equivalent with the projection measure $\nu \cdot \pi^{-1}$, and disintegrate $\nu$ by $\mu$ as follows.

$$
\int_{\Omega \times \mathbf{R}} k(\omega, u) d \nu(\omega, u)=\int_{X} d \mu(x) \int_{\pi(\omega, u)=x} k(\omega, u) d \nu(\omega, u \mid x)
$$

for $k \in L^{1}(\nu) . d \nu(\omega, u \mid x), x \in X$, are sigma-finite, non-atomic and $\widetilde{G}$-invariant measures and satisfy

$$
\nu(\{(\omega, u) \in \Omega \times \mathbf{R} ; \pi(\omega, u) \neq x\} \mid x)=0 \text { a.e. } x \in X .
$$

We obtain a flow $F_{t}$ of $T_{t}$ on $(X, \mu)$ defined by

$$
F_{t}(\pi(\omega, u))=T_{t}(\omega, u)
$$

and call it the associated flow of $G$ [5]. It is known that the isomorphism class of this flow is a complete invariant for the orbit equivalence of $G$ when the action by $G$ is amenable [9].

Definition 9. Let $\Gamma$ be a countable dense subgroup of $\mathbf{R}$. Define the countable non-singular transformation group $\mathscr{G}$ on $(\Omega \times \mathbf{R}$, $\mathscr{B} \otimes \mathscr{B}(\mathbf{R}), \nu)$ by

$$
\mathscr{G}=\left\{\tilde{g} \cdot T_{\gamma} ; g \in G, \gamma \in \Gamma\right\} .
$$

We notice that the associated flow of $\mathscr{G}$ is just that of $G$. Since every $\tilde{g}$ commutes with all $T_{\gamma}, \gamma \in \Gamma$, the action by $\mathscr{G}$ is amenable if 
that of $G$ is amenable. Krieger's theorem ([9]) says that if the action by $G$ is amenable and of type $\mathrm{III}_{0}$ then $G$ is orbit equivalent with $\mathscr{G}$ (see [4]).

Definition 10. For each $h \in[\mathscr{G}]_{*}^{\nu}$ we define the non-negative, integrable function $f_{h} \in \mathbf{L}^{1}(X, \mu)$ and the finite measure $\psi_{h}$ on $\Omega \times \mathbf{R}$ by

$$
\begin{aligned}
f_{h}(x) & =\nu(\mathscr{I}(h) \mid x), \quad x \in X, \\
\psi_{h}(E) & =\nu(h E), \quad \text { for all measurable sets } E \subset \mathscr{D}(h),
\end{aligned}
$$

and in particular we write for $h=\left.\mathrm{id}\right|_{E} \in[\mathscr{G}]_{*}^{\nu}$,

$$
f_{E}(x)=f_{\left.\mathrm{id}\right|_{E}}(x)=\nu(E \mid x), \quad x \in X .
$$

Obviously we have $\left\|f_{E}\right\|_{\mathbf{L}^{1}}=\nu(E)$.

LEMMA 11. (1) The map $E \mapsto f_{E}$ induces a bijection from the $\widetilde{G}$-Hopf equivalence classes of sets $E \in \mathscr{B}$ with $\nu(E)<\infty$ onto $\mathbf{L}^{1}(X, \mu)_{+}$, and this bijection is additive.

(2) $\left\|f_{E}-f_{E^{\prime}}\right\| \leq \nu\left(E \Delta E^{\prime}\right)$.

Proof. (1) It is obvious that for $E$ and $E^{\prime} \in \mathscr{B}$ with $\nu(E)<\infty$, and $\nu\left(E^{\prime}\right)<\infty$, the sets $E$ and $E^{\prime}$ are $\widetilde{G}$-Hopf equivalent if and only if $\nu(E \mid x)=\nu\left(E^{\prime} \mid x\right)$ a.e. $x$. Since each $\nu(\cdot \mid x)$ is an infinite and sigma-finite measure, the map $E \mapsto f_{E} \in \mathbf{L}^{1}(X, \mu)_{+}$is onto. The additivity, that is, $f_{E \cup F}=f_{E}+f_{F}$ for disjoint sets $E$ and $F$, is obvious.

$$
\begin{aligned}
\| f_{E} & -f_{E^{\prime}} \|_{\mathbf{L}^{1}} \\
& =\int_{X} d \mu(x)\left|\int_{\pi(\omega, u)=x}\left\{I_{E}(\omega, u)-I_{E^{\prime}}(\omega, u)\right\} d \nu(\omega, u \mid x)\right| \\
& \leq \int_{X} \nu\left(E \Delta E^{\prime} \mid x\right) d \mu(x) \\
& =\nu\left(E \Delta E^{\prime}\right) .
\end{aligned}
$$

Here $I_{E}$ means the indicator function of a set $E$.

LEMMA 12.

$$
f_{T_{\gamma}^{-1} \cdot h}(x)=\exp (-\gamma) f_{h}\left(F_{\gamma} x\right) \frac{d \mu F_{\gamma}}{d \mu}(x), \quad \text { for } h \in[\mathscr{G}]_{*}^{\nu}, \quad \gamma \in \Gamma .
$$


Proof. For $g \in \mathbf{L}^{\infty}(X, \mu)$,

$$
\begin{aligned}
\int_{X} g & (x) f_{T_{\gamma}^{-1} \cdot h}(x) d \mu(x) \\
& =\int_{X} g(x) d \mu(x) \int_{\pi(\omega, u)=x} I_{T_{\gamma}^{-1}(\mathcal{I}(h))}(\omega, u) d \nu(\omega, u \mid x) \\
& =\int_{\Omega} d m(\omega) \int_{\mathbf{R}} g(\pi(\omega, u)) I_{\mathcal{F}(h)}(\omega, u+\gamma) \exp (u) d u \\
& =\exp (-\gamma) \int_{\Omega} d m(\omega) \int_{\mathbf{R}} g(\pi(\omega, u-\gamma)) I_{\mathscr{I}(h)}(\omega, u) \exp (u) d u \\
& =\exp (-\gamma) \int_{X} g \cdot F_{-\gamma}(x) d \mu(x) \int_{\pi(\omega, u)=x} I_{\mathscr{F}(h)}(\omega, u) d \nu(\omega, u \mid x) \\
& =\int_{X} g(x) \exp (-\gamma) \frac{d \mu F_{\gamma}}{d \mu}(x) f_{h}\left(F_{\gamma} x\right) d \mu(x) .
\end{aligned}
$$

LEMmA 13. Let $\varepsilon>0, h \in[\mathscr{G}]_{*}^{\nu}$ and $f \in \mathbf{L}^{1}(X, \mu)_{+}$. Then there exists a partial transformation $h_{1} \in[\mathscr{G}]_{*}^{\nu}$ satisfying

$$
\left\{\begin{array}{l}
\mathscr{D}\left(h_{1}\right)=\mathscr{D}(h), \\
f_{h_{1}}=f, \quad \text { and } \\
\left\|\psi_{h_{1}}-\psi_{h}\right\| \leq\left\|f-f_{h}\right\|+\varepsilon .
\end{array}\right.
$$

Proof. Decompose the space $X$ into the disjoint subsets $X_{-}, X_{0}$, $X_{+}$defined by

$$
\left\{\begin{array}{l}
X_{-}=\left\{x \in X ; f(x)<f_{h}(x)\right\}, \\
X_{0}=\left\{x \in X ; f(x)=f_{h}(x)\right\}, \\
X_{+}=\left\{x \in X ; f(x)>f_{h}(x)\right\} .
\end{array}\right.
$$

When $\mu\left(X_{+}\right)=\mu\left(X_{-}\right)=0$, set $h_{1}=h$. Otherwise, we may assume $\mu\left(X_{-}\right)>0$. (If $\mu\left(X_{+}\right)>0$ then the proof is parallel.) Since $\nu(\cdot \mid x)$ is an infinite, non-atomic and sigma-finite measure, one can choose a measurable subset $E \subset \Omega \times \mathbf{R}$ such that

$$
\left\{\begin{array}{l}
\nu(E \mid x)=f(x) \quad \text { a.e. } x, \\
E \cap \pi^{-1}\left(X_{-}\right) \subset \mathscr{I}(h) \cap \pi^{-1}\left(X_{-}\right), \\
E \cap \pi^{-1}\left(X_{0}\right)=\mathscr{I}(h) \cap \pi^{-1}\left(X_{0}\right) \text { and }, \\
E \cap \pi^{-1}\left(X_{+}\right) \supset \mathscr{I}(h) \cap \pi^{-}\left(X_{+}\right) .
\end{array}\right.
$$

For the same reason as above, one can also choose measurable subsets 
$E^{\prime} \subset E$ and $F \subset \Omega \times \mathbf{R}$ satisfying

$$
\begin{gathered}
E^{\prime} \cap \pi^{-1}\left(X_{+} \cup X_{0}\right)=E \cap \pi^{-1}\left(X_{+} \cup X_{0}\right), \\
\nu\left(E^{\prime} \mid x\right)<f(x) \quad \text { a.e. } x \in X_{-}, \\
\left\|f(x)-\nu\left(E^{\prime} \mid x\right)\right\|_{\mathbf{L}^{1}(X)}<\frac{\varepsilon}{2}, \\
F \cap \mathscr{I}(h)=\varnothing \text { and } \\
\nu(F \mid x)=\nu(E \mid x)-\nu\left(E^{\prime} \mid x\right) \text { a.e. } x .
\end{gathered}
$$

Since $\mathscr{G}$ is of type III, one can obtain a partial transformation $u \in$ $[\mathscr{G}]_{*}^{\nu}$ such that

$$
\mathscr{D}(u)=\mathscr{I}(h) \backslash E^{\prime}, \quad \mathscr{I}(u)=F .
$$

Noticing $E^{\prime} \cap F=\varnothing$, one can extend $u$ from $\mathscr{I}(h) \backslash E^{\prime}$ to $\mathscr{I}(h)$ by

$$
u(\omega, t)= \begin{cases}(\omega, t), & \text { if }(\omega, t) \in E^{\prime}, \\ u(\omega, t), & \text { if }(\omega, t) \in \mathscr{I}(h) \backslash E^{\prime} .\end{cases}
$$

Then $\mathscr{D}(u)=\mathscr{I}(h)$ and $\mathscr{I}(u)=F \cup E^{\prime}$.

Putting

$$
h_{1}=u \cdot h \in[\mathscr{G}]_{*}^{\nu},
$$

we will check that $h_{1}$ satisfies the condition of the lemma. Obviously, $\mathscr{D}\left(h_{1}\right)=\mathscr{D}(h)$ and $f_{h_{1}}(x)=f(x)$.

$$
\begin{aligned}
\| \psi_{h_{1}} & -\psi_{h} \| \\
& =\|\nu(u h \cdot)-\nu(h \cdot)\| \\
& =\left\|\nu(u \cdot)-\nu\left(\left.\mathrm{id}\right|_{\mathcal{I}(h)}\right)\right\| \\
& =\left\|\nu\left(\left.u\right|_{\left(\mathscr{I}(h) \backslash E^{\prime}\right) \cap \pi^{-1}\left(X_{-}\right)}\right)-\nu\left(\left(\mathscr{I}(h) \backslash E^{\prime}\right) \cap \pi^{-1}\left(X_{-}\right) \cap \cdot\right)\right\|
\end{aligned}
$$

(because $\mathscr{D}(u)=\left\{\left(\mathscr{I}(h) \backslash E^{\prime}\right) \cap \pi^{-1}\left(X_{-}\right)\right\} \cup E^{\prime}=\mathscr{I}(h)$ and $u=$ id on $E^{\prime}$ ).

$$
\begin{aligned}
& \leq \nu\left(u\left(\left(\mathscr{I}(h) \backslash E^{\prime}\right) \cap \pi^{-1}\left(X_{-}\right)\right)\right)+\nu\left(\left(\mathscr{I}(h) \backslash E^{\prime}\right) \cap \pi^{-1}\left(X_{-}\right)\right) \\
& =\int_{X_{-}} \nu(F \mid x) d \mu(x)+\int_{X_{-}} \nu\left(\mathscr{I}(h) \backslash E^{\prime} \mid x\right) d \mu(x) \\
& =\int_{X_{-}}\left\{f(x)-\nu\left(E^{\prime} \mid x\right)\right\} d \mu(x)+\int_{X_{-}}\left\{f(x)-\nu\left(E^{\prime} \mid x\right)\right\} d \mu(x) \\
& <\left\|f(x)-\nu\left(E^{\prime} \mid x\right)\right\|+\left\|f_{h}(x)-\nu\left(E^{\prime} \mid x\right)\right\| \\
& <\frac{\varepsilon}{2}+\left\|f_{h}-f\right\|+\left\|f(x)-\nu\left(E^{\prime} \mid x\right)\right\| \\
& <\varepsilon+\left\|f_{h}-f\right\| .
\end{aligned}
$$


LEMMA 14. If $f_{h}=\sum_{i=1}^{N} f_{i}\left(f_{i} \in \mathbf{L}^{1}(X, \mu)_{+}, h \in[\mathscr{G}]_{*}^{\nu}\right)$ then there exist partial transformations $h_{i} \in[\mathscr{G}]_{*}^{\nu}$ satisfying

$$
\left\{\begin{array}{l}
\mathscr{D}(h)=\bigcup_{i=1}^{N} \mathscr{D}\left(h_{i}\right) \quad \text { (disjoint union) } \\
f_{i}=f_{h_{i}} \text { and } \\
\psi_{h}=\sum_{i=1}^{N} \psi_{h_{i}} .
\end{array}\right.
$$

Proof. Decompose the set $\mathscr{I}(h)$ into a finite number of disjoint measurable sets $\left\{E_{i} ; 1 \leq i \leq N\right\}$ such that

$$
\nu\left(E_{i} \mid x\right)=f_{i}(x) \text { a.e. } X,
$$

and define the partial transformations $h_{i} \in[\mathscr{G}]_{*}^{\nu}$ by

$$
h_{i}(\omega, u)=h(\omega, u), \quad(\omega, u) \in h^{-1} E_{i} .
$$

Then it is easy to check that they satisfy the condition in the lemma.

LEMMA 15. $[\widetilde{G}]_{*}=\left\{h \in[\mathscr{G}]_{*} ; \nu(h \cdot)=\nu(\cdot)\right.$ on $\left.\mathscr{D}(h)\right\}$.

Proof. It is enough to show that if $h \in[\mathscr{G}]_{*}$ is $\nu$-preserving then $h \in[\widetilde{G}]_{*}$. Since for a.e. $(\omega, u) \in \mathscr{D}(h)$,

$$
h(\omega, u)=\tilde{g} \cdot T_{\gamma}(\omega, u)
$$

for some $g \in G$ and $\gamma \in \Gamma$, and since

$$
\frac{d \nu \tilde{g} \cdot T_{\gamma}}{d \nu}(\omega, u)=\exp (\gamma),
$$

$\nu(h \cdot)=\nu(\cdot)$ implies $\gamma=0$ and hence $h(\omega, u)=\tilde{g}(\omega, u)$.

LemMa 16. Let $h, h^{\prime} \in[\mathscr{G}]_{*}^{\nu}$. The following conditions are all equivalent:

(a) $\mathscr{D}(v)=\mathscr{D}\left(h^{\prime}\right), \mathscr{I}(v)=\mathscr{D}(h)$ and $\psi_{h}(v \cdot)=\psi_{h^{\prime}}(\cdot)$ for some $v \in[\mathscr{G}]_{*}^{\nu}$.

(b) The sets $\mathscr{I}(h)$ and $\mathscr{I}\left(h^{\prime}\right)$ are $\widetilde{G}$-Hopf equivalent.

(c) $f_{h}=f_{h^{\prime}}$.

Proof. (a) $\Rightarrow$ (b). Putting $g=h v h^{\prime-1} \in[\mathscr{G}]_{*}^{\nu}$ then

$$
\nu(g \cdot)=\psi_{h}\left(v h^{\prime-1} \cdot\right)=\psi_{h^{\prime}}\left(h^{\prime-1} \cdot\right)=\nu(\cdot) .
$$

By Lemma $15, g \in[\widetilde{G}]_{*}$. 
(b) $\Rightarrow$ (a). If $g \in[\widetilde{G}]_{*}$ satisfies $\mathscr{D}(g)=\mathscr{I}\left(h^{\prime}\right)$ and $\mathscr{I}(g)=$ $\mathscr{I}(h)$, then putting $v=h^{-1} g h^{\prime} \in[\mathscr{G}]_{*}^{\nu}$, we have $\mathscr{D}(v)=\mathscr{D}\left(h^{\prime}\right)$, $\mathscr{I}(v)=\mathscr{D}(h)$ and

$$
\psi_{h^{\prime}}\left(v^{-1} E\right)=\nu\left(h^{\prime} v^{-1} E\right)=\nu\left(g^{-1} h E\right)=\nu(h E)=\psi_{h}(E) .
$$

The equivalence between (b) and (c) was proved in Lemma 11.

4. Measure theoretical proof of the Connes-Woods theorem. Let us recall the definition of an approximately transitive flow (which we briefly call the AT-flow) [2].

Definition 17. A non-singular flow $\left(F_{t}\right)_{t \in \mathbf{R}}$ is said to be approximately transitive (AT) if for any $\theta>0$, and for any finite number of functions $f_{1}, \ldots, f_{n} \in \mathbf{L}^{1}(X, \mu)_{+}$there exist a function $f \in$ $\mathbf{L}^{1}(X, \mu)_{+}$and a finite number of $r(i, l) \in \mathbf{R}, 1 \leq l \leq L_{i}$, such that

$$
\left\|f_{i}-\sum_{l=1}^{L_{i}} \exp (-r(i, l)) f \circ F_{r(i, l)} \frac{d \mu F_{r(i, l)}}{d \mu}\right\|<\theta, \quad 1 \leq i \leq n .
$$

As explained in the introduction, the A. Connes-J. Woods theorem on a characterization of ITPFI-factors can be stated in our setting as follows.

THEOREM 18. Let $G$ be a type III $_{0}$ ergodic amenable action on $(\Omega, \mathscr{B}, m)$, with associated flow $\left(F_{t}\right)_{t \in \mathbf{R}}$. Then $G$ is orbit equivalent with a product odometer action with a product measure if and only if $\left(F_{t}\right)_{t \in \mathbf{R}}$ is conservative, aperiodic, and $A T$.

Now we will give a measure theoretical proof of the only if part of their theorem. Since $G$ is orbit equivalent with $\mathscr{G}$, it is enough to show that $\mathscr{G}$ is orbit equivalent with a product odometer action with a product measure. In fact under the AT-condition, we will check the sufficient condition in Proposition 7 for $\mathscr{G}$ to be orbit equivalent with a product odometer action with a product measure.

Let $\sum_{i=1}^{n} \bigoplus \zeta_{i} \quad\left(\zeta_{i}=\left\{e_{\alpha, \beta} ; \alpha, \beta \in \Lambda_{i}\right\}\right)$ be an arbitrary multiple tower for $\mathscr{G}$ with constant $P$-Jacobian, where $P \sim m$. Take an arbitrary floor $E_{\alpha(i)}$ from each $\zeta_{i}$.

We may assume $P=\nu$ on each $E_{\alpha(i)}$. To see this, for any $\delta>0$ we decompose the sets $E_{\alpha(i)}$ into a finite number of disjoint sets $A_{i, j}$ 
$(0 \leq j \leq J)$ such that

$$
\left\{\begin{array}{l}
c_{i, j} \exp (-\delta)<\frac{d P}{d \nu}(z)<c_{i, j} \exp (\delta), \quad \text { for } z \in A_{i, j}, 1 \leq j \leq J, \\
P\left(A_{i, 0}\right)<\delta \\
\nu\left(A_{i, 0}\right)<\delta
\end{array}\right.
$$

Taking $\zeta_{i}$-invariant sets $\operatorname{Orb}_{\zeta_{l}}\left(A_{i, j}\right)$ and restricting towers $\zeta_{i}$ on these sets we get towers $\zeta_{i, j}$. Let $\mathbf{q}_{i}$ be the distribution of $\zeta_{i}$ relative to $P$. Then if the above $\delta$ is chosen sufficiently small, $\left\|\sum_{i} \nu_{\mathbf{q}_{i}}-P\right\|$ is small. Therefore we may and do replace $\sum_{i} \oplus \zeta_{i}$ by $\sum_{i, j} \oplus \zeta_{i, j}$ and $P$ by $\sum_{i} \nu_{\mathbf{q}_{i}}$ respectively.

We will show the existence of a tower $\xi$ satisfying the following conditions:

(a) $\xi$ refines $\sum_{i, j} \oplus \zeta_{i, j}$.

(b) $\xi$ has constant $Q$-Jacobian, where $Q$ is a finite measure equivalent to $\nu$ such that

$$
\left\|Q-\nu_{\mathbf{q}}\right\|_{\left(\sum_{i} \oplus \zeta_{\imath}\right)}<\delta^{\prime}
$$

Here $\mathbf{q}$ is the distribution of $\sum_{i=1}^{n} \bigoplus \zeta_{i}$ relative to $P$ and $\delta^{\prime}>0$ satisfies

$$
\sum_{i, j} c_{i, j} \exp (\delta) \delta^{\prime}<\theta-2 \delta .
$$

Construct the (uniquely determined) measure $Q^{\prime}$ satisfying the following conditions:

(a) $Q^{\prime}(E)=c_{i, j} Q(E)$, for all measurable subsets $E \subset A_{i, j}, j \geq 1$,

(b) $Q^{\prime}(E)=Q(E)$, for all measurable subsets $E \subset A_{i, 0}$,

(c) both of $Q$ and $Q^{\prime}$-distributions of $\sum_{i} \oplus \zeta_{i}$ coincide.

Then we get

$$
\left\|Q^{\prime}-P\right\|_{\bigcup_{t=1}^{n} s\left(\zeta_{\iota}\right)}<\theta
$$

and by Proposition 7, the transformation group $\mathscr{G}$ will be orbit equivalent with a product odometer action with a product measure. Therefore in order to complete the proof, it is enough to construct a tower $\xi$ and a measure $Q$ satisfying (a), (b).

Since for any $f \in \mathbf{L}^{1}(X, \mu)$, the map

$$
t \in \mathbf{R} \mapsto f \circ F_{t} \frac{d \mu F_{t}}{d \mu}(x) \in \mathbf{L}^{1}(X, \nu)
$$


is continuous and since $\Gamma$ is dense, $\left(F_{\gamma}\right)_{\gamma \in \Gamma}$ is AT, too. That is, there exist $f \in \mathbf{L}^{1}(X, \mu)_{+}$and a finite number of $\gamma(i, l) \in \Gamma, 1 \leq i \leq n$, $1 \leq l \leq L_{i}$ such that

$$
\left\|f_{E_{\alpha(l)}}-\sum_{l=1}^{L_{l}} \exp (-\gamma(i, l)) f \circ F_{\gamma(i, l)} \frac{d \mu F_{\gamma(i, l)}}{d \mu}\right\|<\theta,
$$

$1 \leq i \leq n$.

Next applying Lemma 13 for the partial transformation id $\left.\right|_{E_{\alpha(l)}} \in[\mathscr{G}]_{*}^{\nu}$ and the function

$$
\sum_{l=1}^{L_{l}} \exp (-\gamma(i, l)) f \circ F_{\gamma(i, l)} \frac{d \mu F_{\gamma(i, l)}}{d \mu} \in \mathbf{L}^{1}(X, \mu)_{+}
$$

we obtain a partial transformation $h_{i} \in[\mathscr{G}]_{*}^{\nu}$ such that

$$
\begin{aligned}
\mathscr{D}\left(h_{i}\right) & =E_{\alpha(i)}, \\
f_{h_{l}} & =\sum_{l} \exp (-\gamma(i, l)) f \circ F_{\gamma(i, l)} \frac{d \mu F_{\gamma(i, l)}}{d \mu}
\end{aligned}
$$

and

$$
\begin{aligned}
& \left\|\psi_{h_{l}}(\cdot)-\nu\left(E_{\alpha(i)} \cap \cdot\right)\right\| \\
& \leq\left\|f_{E_{\alpha(l)}}-\sum_{l} \exp (-\gamma(i, l)) f \circ F_{\gamma(i, l)} \frac{d \mu F_{\gamma(i, l)}}{d \mu}\right\|+\theta<2 \theta, \\
& 1 \leq i \leq n \quad \text { (use (3)). }
\end{aligned}
$$

Since $f_{h_{\imath}}$ is a finite sum of $\mathbf{L}^{1}(X, \mu)_{+}$-functions

$$
\exp (-\gamma(i, l)) f\left(F_{\gamma(i, l)} x\right) \frac{d \mu F_{\gamma(i, l)}}{d \mu}(x),
$$

it follows from Lemma 14 that there exist partial transformations $h_{l}^{i} \in[\mathscr{G}]_{*}^{\nu}$ satisfying

$$
\begin{aligned}
E_{\alpha(i)} & =\bigcup_{l=1}^{L_{i}} \mathscr{D}\left(h_{l}^{i}\right) \quad \text { (disjoint union) } \\
f_{h_{l}^{l}}(x) & =\exp (-\gamma(i, l)) f\left(F_{\gamma(i, l)} x\right) \frac{d \mu F_{\gamma(i, l)}}{d \mu}(x), \quad \text { and } \\
\psi_{h_{\imath}} & =\sum_{l} \psi_{h_{l}^{\prime}}
\end{aligned}
$$


Then,

$$
\begin{aligned}
f_{h_{l}^{l}}(x)= & \exp (-\gamma(i, l)) f\left(F_{\gamma(i, l)} x\right) \frac{d \mu F_{\gamma(i, l)}}{d \mu}(x) \\
= & \exp (-\gamma(i, l)) f\left(F_{\gamma(1,1)}\left(F_{\gamma(i, l)-\gamma(1,1)} x\right)\right) \\
& \cdot \frac{d \mu F_{\gamma(1,1)}}{d \mu}\left(F_{\gamma(i, l)-\gamma(1,1)} x\right) \frac{d \mu F_{\gamma(i, l)-\gamma(1,1)}}{d \mu}(x) \\
= & \exp (-\gamma(i, l)+\gamma(1,1)) f_{h_{1}^{1}}\left(F_{\gamma(i, l)-\gamma(1,1)} x\right) \frac{d \mu F_{\gamma(i, l)-\gamma(1,1)}}{d \mu}(x) \\
= & f_{T_{\gamma(i, l)-\gamma(1,1)}^{-1} \cdot h_{1}^{1}} \quad \text { (use Lemma 12). }
\end{aligned}
$$

Applying Lemma 16 for partial transformations $h_{l}^{i}$ and $T_{\gamma(i, l)-\gamma(1,1)}{ }^{*}$ $h_{1}^{1} \in[\mathscr{G}]_{*}^{\nu}$ we obtain partial transformations $v_{l}^{i} \in[\mathscr{G}]_{*}^{\nu}$ satisfying

$$
\left\{\begin{array}{l}
\mathscr{D}\left(v_{l}^{i}\right)=\mathscr{D}\left(h_{1}^{1}\right) \\
\mathscr{I}\left(v_{l}^{i}\right)=\mathscr{D}\left(h_{l}^{i}\right) \\
\psi_{h_{l}^{l}}\left(v_{l}^{i} \cdot\right)=\psi_{T_{\gamma(t, l)-\gamma(1,1)}^{-1} \cdot h_{1}^{1}}(\cdot)
\end{array}\right.
$$

Since the flow $\left(T_{t}\right)_{t \in \mathbf{R}}$ scales down the $\widetilde{G}$-invariant measure $\nu$, we get

$$
\psi_{T_{\gamma(1, l) \gamma(1,1)}^{-1} \cdot h_{1}^{1}}(\cdot)=\exp (-\gamma(i, l)+\gamma(1,1)) \nu\left(h_{1}^{1} \cdot\right) .
$$

Therefore, we get

$$
\nu\left(h_{l}^{i} v_{l}^{i} \cdot\right)=\exp (-\gamma(i, l)+\gamma(1,1)) \nu\left(h_{1}^{1} \cdot\right) .
$$

Finally we construct the measure $Q$ by

$$
Q\left(e_{\beta, \alpha(i)} E\right)=\frac{P\left(E_{\beta}\right)}{P\left(E_{\alpha(i)}\right)} \nu\left(h_{l}^{i} E\right), \quad \text { for } E \subset \mathscr{D}\left(h_{l}^{i}\right) .
$$

Here $E_{\beta}$ is a floor of the tower $\zeta_{i}$. Then one can see that

$$
\begin{aligned}
& \left\|Q\left(E_{\alpha(i)} \cap \cdot\right)-\nu\left(E_{\alpha(i)} \cap \cdot\right)\right\|_{E_{\alpha(l)}} \\
& \quad=\left\|\sum_{l} \psi_{h_{l}^{l}}(\cdot)-\nu\left(E_{\alpha(i)} \cap \cdot\right)\right\|_{E_{\alpha(l)}} \\
& \quad=\left\|\psi_{h_{l}}(\cdot)-\nu\left(E_{\alpha(i)} \cap \cdot\right)\right\|_{E_{\alpha(l)}} \\
& \quad<2 \theta
\end{aligned}
$$


and that

$$
\begin{aligned}
\frac{d Q v_{l}^{i}}{d Q}(z) & =\frac{d \nu h_{l}^{i} v_{l}^{i}}{d \nu h_{1}^{1}}(z) \\
& =\exp (-\gamma(i, l)+\gamma(1,1)), \quad z \in \mathscr{D}\left(h_{1}^{1}\right) .
\end{aligned}
$$

Defining the partial transformations $e_{i \alpha r, j \beta s}$ for $1 \leq r \leq L_{i}, 1 \leq s \leq$ $L_{j}, \alpha \in \Lambda_{i}$, and $\beta \in \Lambda_{j}$, by

$$
\begin{aligned}
e_{i \alpha r, j \beta s}(z)= & e_{\alpha, \alpha(i)} v_{r}^{i}\left(v_{s}^{j}\right)^{-1} e_{\alpha(j), \beta}(z) \\
& \text { if } z \in E_{\beta}\left(\text { a floor of } \zeta_{j}\right), \quad \text { and } e_{\alpha(j), \beta} z \in \mathscr{D}\left(h_{s}^{j}\right),
\end{aligned}
$$

we obtain a tower $\xi=\left\{e_{i \alpha r, j \beta s} ; 1 \leq i, j \leq n, 1 \leq r \leq L_{i}, 1 \leq\right.$ $s \leq L_{j}, \alpha \in \Lambda_{i}$, and $\left.\beta \in \Lambda_{j}\right\}$ which refines the multiple tower $\sum_{i, j} \bigoplus \zeta_{i, j}$ and has a constant $Q$-Jacobian. Of course, the estimate (4) is enough for that of $\left\|Q-\nu_{\mathbf{q}}\right\|_{s\left(\sum_{t} \oplus \zeta_{\imath}\right)}$ in (2), since each $e_{\alpha, \alpha(i)}$ is non-singular and $P=\nu$ on each $E_{\alpha(i)}$.

Corollary 19. The sufficient condition in Proposition 7 for an ergodic countable group of non-singular transformations of type $\mathrm{III}_{0}$ to be orbit equivalent with a product odometer action with a product measure is a necessary condition.

Proof. The associated flow of a product odometer action with a product measure is AT. We have already checked in the proof of Theorem 18 that the condition (b) of Proposition 7 holds under the assumption that the associated flow is AT.

\section{REFERENCES}

[1] H. Araki and E. J. Woods, A classification of factors, Publ. Res. Inst. Math. Sci., 4 (1968), 51-130.

[2] A. Connes and E. J. Woods, Approximately transitive flows and ITPFI factors, Ergodic Theory Dynamical Systems, 5 (1985), 203-236.

[3] A. Connes, J. Feldman and B. Weiss, Amenable equivalence relations are hyperfinite, Ergodic Theory Dynamical Systems, 1 (1981), 431-450.

[4] T. Hamachi, The normalizer group of an ergodic automorphism of type III and the commutant of an ergodic flow, J. Funct. Anal., 40 (1981), 387-403.

[5] T. Hamachi and M. Osikawa, Ergodic groups of automorphisms and Krieger's theorems, Seminar on Mathematical Science of Keio Univ., 3 (1981), 1-113.

[6] J. M. Hawkins, Properties of ergodic flows associated with product odometers, Pacific J. Math., 141 (1990), 287-294.

[7] Y. Katznelson, Lectures on orbit equivalence, mimeographed notes, Orsay, 1980.

[8] W. Krieger, On non-singular transformations of a measure space I, II, Z. Wahrscheinlichkeitstheorie verw., 11 (1969), 83-97, 98-119. 
[9] W. Krieger, On ergodic flows and isomorphism of factors, Math. Ann., 223 (1976), 19-70.

[10] E. Størmer, Hyperfinite product factors, I, II, III, Arkiv für Mathematik, 9 (1971), 165-170; J. Funct. Anal., 10 (1972), 471-481; Amer. J. Math., 97 (1975), 589-595.

Received November 20, 1990 and in revised form June 18, 1991.

College of General Education

KYUSHU UNIVERSITY

Ropponmatsu, ChUO-KU

FUKUOKA 810, JAPAN 



\section{PACIFIC JOURNAL OF MATHEMATICS EDITORS}

V. S. VARAdARAJAN

(Managing Editor)

University of California

Los Angeles, CA 90024-1555

Herbert Clemens

University of Utah

Salt Lake City, UT 84112

F. Michael CHRIST

University of California

Los Angeles, CA 90024-1555

ThOMAs ENRIGHT

University of California, San Diego

La Jolla, CA 92093
Nicholas ERCOLANI

University of Arizona

Tucson, AZ 85721

R. FINN

Stanford University

Stanford, CA 94305

VAUGHAN F. R. JoNES

University of California

Berkeley, CA 94720

SteVen KercKhofF

Stanford University

Stanford, CA 94305
C. C. MOORE

University of California

Berkeley, CA 94720

MARTIN SCHARLEMANN

University of California

Santa Barbara, CA 93106

HAROLD STARK

University of California, San Diego

La Jolla, CA 92093

\section{ASSOCIATE EDITORS}
R. Arens
E. F. BeCKenBACH
B. H. NeumanN
F. WolF
(1904-1989)
K. YoshidA (1906-1982)

TIONS

UNIVERSITY OF ARIZONA

UNIVERSITY OF BRITISH COLUMBIA

UNIVERSITY OF OREGON

UNIVERSITY OF SOUTHERN CALIFORNIA

CALIFORNIA INSTITUTE OF TECHNOLOGY

UNIVERSITY OF CALIFORNIA

MONTANA STATE UNIVERSITY

STANFORD UNIVERSITY

UNIVERSITY OF HAWAII

UNIVERSITY OF NEVADA, RENO

UNIVERSITY OF TOKYO

NEW MEXICO STATE UNIVERSITY

UNIVERSITY OF UTAH

OREGON STATE UNIVERSITY

WASHINGTON STATE UNIVERSITY

UNIVERSITY OF WASHINGTON 


\section{Pacific Journal of Mathematics}

Vol. 154, No. $1 \quad$ May, 1992

Richard Arens, Pseudo regular elements in a normed ring $\ldots \ldots \ldots \ldots \ldots 1$

Joan Birman and William W. Menasco, Studying links via closed braids.

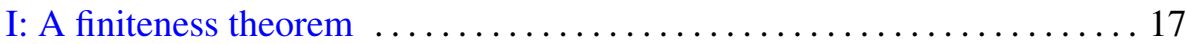

Etsurō Date, Michio Jimbo, Kei Miki and Tetsuji Miwa, Braid group

representations arising from the generalized chiral Potts models ....... 37

Toshihiro Hamachi, A measure theoretical proof of the Connes-Woods

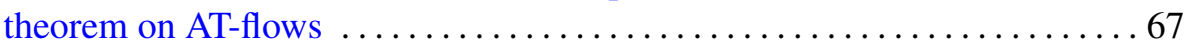

Allen E. Hatcher and Ulrich Oertel, Affine lamination spaces for surfaces ....................................... 87

David Joyner, Simple local trace formulas for unramified $p$-adic groups $\ldots .103$

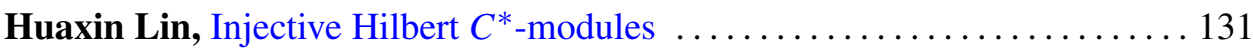

John Marafino, The boundary of a simply connected domain at an inner

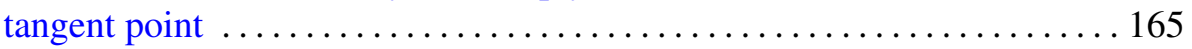

Gonzalo Riera and Rubi Rodriguez, The period matrix of Bring's curve . . 179 